\title{
EFICÁCIA DE ESTRATÉGIAS EDUCATIVAS PARA AÇÕES PREVENTIVAS DA PNEUMONIA ASSOCIADA À VENTILAÇÃO MECÂNICA
}

\author{
Efficacy of health education strategies for preventive interventions of ventilator- \\ associated pneumonia
}

\section{Eficacia de las estrategias educativas para la acción preventiva de neumonía asociada a ventilación mecánica}

Fernanda Alves Ferreira Gonçalves ${ }^{1}$
Carlos Roberto Caixeta $^{4}$
Virginia Visconde Brasil ${ }^{2}$

Lizete Malagoni de Almeida Cavalcante Oliveira ${ }^{5}$
Ruth Minamisava ${ }^{3}$

JacquelineAndréiaBernardesLeão Cordeiro ${ }^{6}$

\section{RESUMO}

Objetivou-se determinar a eficácia de estratégia educativa para melhorar o desempenho da equipe de enfermagem na realização de procedimentos preventivos da pneumonia associada à ventilação mecânica. Ensaio clínico controlado não randomizado, conduzido em 2011, em uma unidade de terapia intensiva de Goiânia-Goiás, com a equipe de enfermagem alocada nos grupos intervenção $(n=24)$ e comparação $(n=11)$. Trinta dias após workshops (intervenção), os grupos foram sistematicamente observados usando-se checklist sobre montagem dos ventiladores, posicionamento da cabeceira, mudança de decúbito, uso de equipamentos de proteção individual, higiene brônquica e oral, verificação da pressão do cuffe instalação da sonda enteral. Cada procedimento foi utilizado como unidade de análise e desfecho. A intervenção foi eficaz para higienização da língua (51\%), montagem do ventilador (43\%) e ordem correta tubo-nariz-boca na higiene brônquica (13\%). Estudos em diferentes cenários poderão confirmar a eficácia da estratégia analisada (workshop), assim como identificar outras estratégias educativas úteis para prevenção da pneumonia associada à ventilação mecânica.

Palavras-chave: Pneumonia associada à ventilação mecânica. Educação continuada. Enfermagem.

\begin{abstract}
The objective of the study was to determine the efficacy of educational strategy to improve the performance of nursing staff in performing preventive procedures of ventilator-associated pneumonia. This randomized controlled clinical trial was conducted during 2011 in an intensive care unit in Goiânia, Brazil, with the nursing staff allocated in the intervention group $(n=24)$ and comparison group $(n=11)$. Thirty days after workshops (intervention), the groups was systematically observed using a checklist including ventilator setting-up, angle for bed head elevation, patient decubitus position changing, use of personal protective equipment, bronchial and oral hygiene, verification of the cuff pressure and installation of enteral feeding. Each procedure was used as a unit of analysis and outcome. The intervention was effective for cleaning the tongue $(51 \%)$, for the ventilator setting-up (43\%) and for the correct order tubenose-mouth during bronchial hygiene (13\%). Studies in different scenarios should confirm the efficacy of studied strategy (workshop), as well as identify additional educational strategies to prevent ventilator-associated pneumonia.
\end{abstract}

Keywords: Pneumonia, Ventilator-Associated. Education, Continuing. Nursing.

\section{Resumen}

El objetivo fue determinar la eficacia de la estrategia educativa para mejorar el desempeño del equipo de enfermería en la realización de procedimientos de prevención de neumonía asociada a la ventilación mecánica. Se llevó a cabo un ensayo clínico controlado no aleatorio en 2011, en una Unidad de Cuidados Intensivos en Goiânia, Goiás, con el equipo de enfermería asignado en el grupo de intervención $(n=24)$ y de comparación $(n=11)$. Treinta días después del workshop (intervención), los grupos fueron observados sistemáticamente por médio de una lista de verificación de la instalación del ventilador, posicionamiento de la cabeza, cambio de la posición, el uso del equipo de protección personal, la higiene bronquial y la verificación por vía oral de la presión del manguito y la instalación de la alimentación enteral. Cada procedimiento se utilizó como unidad de análisis y de resultados. La intervención fue efectiva para la limpieza de la lengua (51\%), la instalación del ventilador $(43 \%)$ y la correcta secuencia de succión tubo-narizboca, en la higiene bronquial (13\%). Estudios en diferentes escenarios podrían confirmar la eficacia de la estrategia educativa (workshop), así como identificar otras estrategias educativas para prevenir la neumonía asociada a la ventilación mecánica.

Palabras clave: Neumonía Asociada al Ventilador. Educación continua. Enfermería.

\footnotetext{
${ }^{1}$ Mestre em Enfermagem. Enfermeira da Unidade de Terapia Intensiva do Hospital das Clínicas da Universidade Federal de Goiás. Goiânia-GO. Brasil. email: mestradofernanda@gmail.com; 2Doutora em Enfermagem. Professor Associado da Faculdade de Enfermagem da Universidade Federal de Goiás. Goiânia-GO. Brasil. e-mail: virginia@fen.ufg.br; ${ }^{3}$ Doutora em Epidemiologia. Professor Associado da Faculdade de Enfermagem da Universidade Federal de Goiás. Goiânia-GO. Brasil. e-mail: ruth@fen.ufg.br ; ${ }^{4}$ Mestre em Enfermagem. Enfermeiro da Unidade de Terapia Intensiva do Hospital das Clínicas da Universidade Federal de Goiás. Goiânia-G0. Brasil. E-mail: caixeta2@uol.com.br ; ${ }^{5}$ Doutora em Ciências da Saúde. Professor Associado da Faculdade de Enfermagem da Universidade Federal de Goiás. Goiânia-GO. Brasil. e-mail: lizete@fen.ufg.br ; ${ }^{6}$ Doutoranda em Enfermagem. Professor Assistente da Faculdade de Enfermagem da Universidade Federal de Goiás. Goiânia-GO. Brasil. e-mail: jackbl@uol.com.br
} 


\section{INTRODUÇÃO}

A pneumonia associada à ventilação mecânica (PAV) é uma das complicações mais comuns nos pacientes ventilados mecanicamente no ambiente de terapia intensiva, elevando a mortalidade, o tempo de internação e os custos hospitalare ${ }^{1-4}$.

0 mecanismo mais comum da PAV está relacionado à perda da proteção das vias aéreas, o que favorece o crescimento de micro-organismos tanto exógenos quanto endógenos. 0 paciente intubado e em ventilação mecânica (VM) por período superior a 48 horas tem de seis a 21 vezes maior risco de desenvolver PAV5. Algumas condições relacionadas com a assistência à saúde são fatores de risco para a PAV, tais como aquelas que aumentam a colonização da orofaringe e/ou estômago por bactérias patogênicas (uso de antimicrobianos, internação em unidades de terapia intensiva), situações que causam a aspiração para o trato respiratório ou refluxo no trato gastrointestinal (intubação ou reintubação orotraqueal, uso de sondas gastrointestinais, permanência da cabeceira da cama elevada a menos de 30 graus, imobilidade devida ao coma, trauma ou cirurgias), uso prolongado da VM e mãos dos profissionais de saúde (PAS) contaminadas ${ }^{4,6}$.

Os fatores de risco citados podem ser reduzidos com medidas centradas em ações da equipe que assiste o indivíduo sob VM, como a higienização das mãos dos PAS, a implantação de protocolos que estimulem o uso racional de antimicrobianos, a interrupção diária da sedação, o desmame ventilatório e, ainda, estratégias que visam à redução de procedimentos invasivos na Unidade de Terapia Intensiva (UTI) ${ }^{6-7}$. Além dessas medidas, também são importantes: a prevenção da colonização do trato gastrointestinal, a manutenção da cabeceira da cama elevada a $30^{\circ}$ ou mais, a higiene oral dos pacientes, a limpeza das vias aéreas e o uso de técnica asséptica para manejo da prótese e circuitos ventilatórios ${ }^{4,6,8,9}$.

Devido à complexidade das ações preventivas da PAV, tem sido preconizado o uso de pacotes de cuidados (bundles), em vez de medidas isoladas de prevenção. Adicionalmente, para uma efetiva implementação de atividades preventivas da PAV, programas educativos têm sido largamente recomendados ${ }^{4,6}$. Essas intervenções educativas têm sido relatadas, mas apresentam resultados variáveis e, nem sempre, permanentes ${ }^{9}$. Todavia, o fracasso de parte das intervenções educativas não reduz sua importância e benefícios na redução do tempo de internação e até mesmo da mortalidade ${ }^{8}$. Por essa razão, é fortemente recomendada na prática clínica a educação continuada, o envolvimento dos membros da equipe, a divulgação da epidemiologia e medidas preventivas da PAV, de acordo com a competência e o nível de responsabilidade de cada profissionala,7.

A responsabilidade da equipe de enfermagem para 0 controle e prevenção da PAV é considerável, pois ela realiza a maioria dos cuidados relativos ao uso de VM na UTR². A carência de evidências fortes sobre quais estratégias educativas são mais adequadas para cada procedimento pode levar à manutenção de programas educacionais ineficientes e, secundariamente, à alta incidência de PAV.

Em uma UTI-escola de Goiânia, Goiás, foi realizada auditoria diária dos cuidados de enfermagem para prevenção da PAV, com piores resultados para os seguintes procedimentos: posição da cabeceira da cama, higiene brônquica, cuidados com os circuitos e condensados do VM, higienização de mãos, cuidados com a umidificação do oxigênio, avaliação do resíduo gástrico, higiene oral e verificação da pressão do cuff três vezes ao dia. 0 resultado desta auditoria também mostrou que nem todos os cuidados para prevenção da PAV eram executados por todos os membros da equipe de enfermagem, indicando a necessidade de implementação de estratégias educativas mais eficazes para esses trabalhadores.

Esse estudo testa o emprego de uma estratégia educativa, concentrada nos tópicos que a auditoria havia revelado como condutas não condizentes com protocolos baseados em evidências. 0 objetivo do presente estudo foi determinar a eficácia de estratégia educativa para melhorar o desempenho de procedimentos preventivos da pneumonia associada à ventilação mecânica.

\section{MÉTODO}

Ensaio clínico controlado não randomizado, conduzido entre fevereiro e agosto de 2011, em uma UTI cirúrgica de uma instituição pública de ensino, com oito leitos, do município de Goiânia/GO, com rotina de auditoria de cuidados de enfermagem relacionados à prevenção da PAV. 0 estudo foi aprovado pelo Comitê de Ética em Pesquisa do Hospital das Clínicas da Universidade Federal de Goiás (CEP/HC/UFG), parecer n 146/2010. Todos os sujeitos foram esclarecidos sobre os objetivos e procedimentos do estudo, e sua anuência foi registrada com a assinatura do Termo de Consentimento Livre e Esclarecido.

Antes de iniciar o estudo, diversos cartazes estilizados com charges haviam sido afixados em locais estratégicos dentro da unidade. Figuras, fotos e textos relacionados ao ambiente de terapia intensiva foram selecionados por um enfermeiro para serem usados nas charges. Os temas dos cartazes eram relacionados à prevenção da PAV.

Os critérios de inclusão foram: ser enfermeiro ou técnico de enfermagem da UTI há mais de seis meses e estar trabalhando na UTI durante o período da coleta de dados. Atenderam aos critérios de inclusão 35 sujeitos dos 38 que trabalhavam na unidade, sendo 7 enfermeiros e 28 técnicos de enfermagem, não tendo havido recusas.

Os participantes do estudo foram alocados nos grupos intervenção e comparação, de acordo com a escolha de cada trabalhador. 0 grupo intervenção participou do programa educativo e o grupo comparação não participou (Figura 1). 


\section{Estratégias educativas para profilaxia da PAV}

Figura1.Fluxograma do estudo

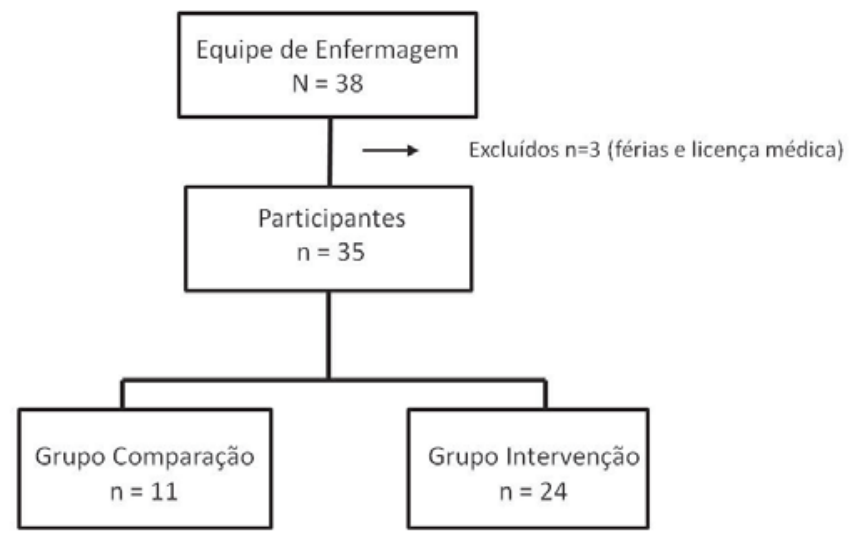

A estratégia educativa usada para incentivar a prevenção da PAV foi o workshop. Ocorreram 12 workshopsno próprio setor de trabalho, com duração de quatro horas cada, durante o horário de trabalho e, com grupo de no máximo 5 profissionais. Participaram do grupo de intervenção 24 sujeitos (68,6\%), sendo 7 enfermeiros $(100,0 \%)$ e 17 técnicos de enfermagem $(70,8 \%)$.

Durante os workshops foram discutidos os cuidados de aspiração traqueal, higiene de mãos, higiene oral, verificação de pressão de cuff, uso de EPI, posicionamento do paciente no leito e cuidados com a instalação e manutenção de dieta enteral. Também foram analisadas as charges dos cartazes. Além disso, o conteúdo dos workshops incluiu: definição e fisiopatologia da PAV; epidemiologia de PAV e seu perfil na UTI cirúrgica; métodos de diagnóstico da PAV; tipos de tratamento; bundles para prevenção de PAV e, ainda, a apresentação e discussão das recomendações do Centers for Disease Control and Prevention $(C D C)^{6}$ e da Agência de Vigilância Sanitária $(\text { ANVISA) })^{4,7}$.

Durante os três meses de intervenção educativa, materiais ainda não existentes na UTI foram adquiridos: equipamentos para verificação da pressão de cuff(manômetros adaptados com sonda de aspiração, torneira de três vias e seringa de $5 \mathrm{~cm}^{3}$ ), caixas inox para armazenamento dos materiais utilizados na higiene oral e dispostas em cada leito; sugadores bucais descartáveis para estimular a troca da sonda de aspiração durante a higiene oral; abridores de boca esterilizáveis com afastador de lábios e bochechas para facilitar o acesso à cavidade bucal e limpadores ou raspadores linguais reprocessáveis para remoção mecânica do biofilme lingual. 0 procedimento de higiene oral foi discutido por mais tempo (duas horas) que os demais temas por solicitação dos participantes do workshop; houve a colaboração de dois cirurgiões-dentistas residentes, e foi acrescida uma atividade prática individualizada, com supervisão.

Durante o período de intervenção, os gestores da unidade, juntamente com o serviço de controle de infecção hospitalar, realizaram nova auditoria na UTI devido ao aumento na incidência da PAV.
Para avaliar os resultados a médio prazo aguardou-se trinta dias após a intervenção educativa para dar início à observação sistematizada dos procedimentos realizados. Essa observação foi feita pela pesquisadora principal por meio de um checklist para registro dos dados. 0 checklist foi construído com base em estudo brasileiro ${ }^{11}$ e adaptado com recomendações do Guidelines for Preventing Health-Care Associated Pneumonia ${ }^{6}$ e do protocolo da ANVISA ${ }^{4}$. 0 checklistincluiu os seguintes procedimentos e passos:

- Ventiladores mecânicos: observados os cuidados antes e durante a montagem da VM, no início do processo ventilatório e ao instalar a nebulização.

- Posicionamento no leito: posicionamento da cabeceirae mudança de decúbito.

- Aspiração de vias aéreas: uso de equipamentos de proteção individual (EPI) e execução da técnica de higiene brônquica.

- Higiene oral: produto usado e execução da técnica de higienização bucal.

- Tubo orotraqueal e traqueostomia: verificação da pressão do cuff.

- Sonda enteral: instalação e testagem da sonda (avaliação clínica e radiológica).

Cada profissional foi observado em dias e horários aleatórios, e foram registrados os passos antes, durante e após o procedimento. Cada passo de cada procedimento foi considerado adequado ou inadequado de acordo com as recomendações citadas acima.

Os dados coletados foram digitados e analisados com auxílio do programa Statistical Package for Social Science (SPSS), versão 11.5 para Windows. Os grupos intervenção e controle foram comparados, e a medida considerada foi a eficácia da intervenção educativa ( 1 - Risco Relativo) para o grupo intervenção. Todas as variáveis foram dicotomizadas. 0 risco relativo foi obtido pelo teste do $x^{2}$. Foram considerados significativamente estatísticos os valores de $p<0,05$ bicaudal e o intervalo de $95 \%$ de confiança (IC95\%), usando o teste de Fisher quando apropriado. 


\section{RESULTADOS}

Houve um total de 3.864 passos de procedimentos, sendo 3.273 no grupo intervenção e 591 no grupo de comparação. As características dos dois grupos foram comparáveis em relação ao sexo, idade, tempo de experiência profissional e tempo de atuação em UTI (Tabela 1).

A maioria do grupo do estudo é do sexo feminino, de enfermeiros jovens e experientes, tanto na profissão quanto no trabalho em ambiente de tratamento intensivo.
Dentre os 17 técnicos de enfermagem, 70,6\% (12/17) têm formação superior em enfermagem, apesar de exercerem a função de técnico, e todos os enfermeiros têm pós-graduação lato ou stricto sensu.

Os cuidados da equipe de enfermagem reconhecidos como efetivos para a profilaxia da pneumonia associada à ventilação mecânica foram analisados entre os grupos de intervenção e comparação (Tabela 2).

Tabela 1. Distribuição de 35 profissionais de enfermagem da Unidade de Terapia Intensiva de um hospital-escola, segundo características sociodemográficas. Goiânia/G0, 2011.

\begin{tabular}{lcc}
\hline \multicolumn{1}{c}{ Variáveis } & $\begin{array}{c}\text { Grupo Intervenção } \\
(\mathbf{n = 2 4 )} \\
\mathbf{n}(\mathbf{\%})\end{array}$ & $\begin{array}{c}\text { Grupo Comparação (n=11) } \\
\mathbf{n}(\mathbf{\%})\end{array}$ \\
\hline $\begin{array}{l}\text { Sexo } \\
\text { Masculino }\end{array}$ & $07(29,2)$ & $02(18,2)$ \\
Feminino & $17(70,8)$ & $09(81,8)$ \\
Idade & $39,2 \pm 6,0$ & $42,1 \pm 6,1$ \\
Idade média \pm desvio-padrão & & $12,4 \pm 5,9$ \\
Experiência Profissional & $14,3 \pm 5,8$ & \\
Média \pm desvio-padrão & & $8,8 \pm 4,6$ \\
Tempo de Atuação em UTI & $8,8 \pm 4,5$ & \\
Média \pm desvio-padrão &
\end{tabular}

Tabela 2. Probabilidade de desempenho adequado dos cuidados da equipe de enfermagem relacionados à profilaxia da pneumonia associada à ventilação mecânica, após programa educativo na Unidade de Terapia Intensiva de um hospital-escola. Goiânia/GO, 2011.

\begin{tabular}{|c|c|c|c|c|c|}
\hline Procedimentos & $\begin{array}{c}\text { Grupo Irterrençāo } \\
\mathrm{n} / \mathrm{N}(\mathbf{\%})\end{array}$ & $\begin{array}{c}\text { Grupo } \\
\text { Comparaçäo } \\
n / \mathbb{N}(\mathbf{\$})\end{array}$ & RR & IC $95 \%$ & $\rho$ \\
\hline $\begin{array}{l}\text { Realiza montagem do ventilador } \\
\text { com técrica asséptica }\end{array}$ & $22 / 41(53,7)$ & $02 / 10(20,0)$ & 0,57 & $(0,36-0,91)$ & $0,048^{*}$ \\
\hline $\begin{array}{l}\text { Descarta condensado do } \\
\text { ventilador mecânico de forma } \\
\text { inadequada }\end{array}$ & $08 / 27(29,6)$ & $10 / 23(43,5)$ & 1,25 & $(0,81-1,92)$ & 0,309 \\
\hline $\begin{array}{l}\text { Usa EPI para descantar o } \\
\text { condensado }\end{array}$ & $21 / 62(33,9)$ & $18 / 33(54,5)$ & 1,45 & $(0,96-2,20)$ & 0,052 \\
\hline Troca nebulizador após o uso & $25 / 63(39.7)$ & $07 / 20(35,0)$ & 0,92 & $(0,63-1,35)$ & 0,707 \\
\hline Reaiza mudança de decibito & $382 / 534(71,5)$ & $178 / 258(69.0)$ & 0,91 & $(0,73-1,15)$ & 0,462 \\
\hline $\begin{array}{l}\text { Mantém ângulo da cabeceira da } \\
\text { cama major que } 30 \text { graus }\end{array}$ & $81 / 102(79,4)$ & $47 / 59(79,7)$ & 1,01 & $(0,54-1,91)$ & 0,997 \\
\hline Higjeniza a lingua & $34 / 49(69,4)$ & $05 / 13(38.5)$ & 0,49 & $(0,27-0,90)$ & $0,043^{*}$ \\
\hline $\begin{array}{l}\text { Usa dorexioina após higiene } \\
\text { bucal }\end{array}$ & $28 / 49(57,1)$ & $06 / 13(46,2)$ & 0,79 & $(0,43-1,45)$ & 0,480 \\
\hline $\begin{array}{l}\text { Verifica pressäo cuff antes do } \\
\text { procedimento de higjene bucal }\end{array}$ & $05 / 49(10,2)$ & $0 / 13(0,0)$ & 0,89 & $(0,82-0,99)$ & $0,574^{*}$ \\
\hline $\begin{array}{l}\text { Realiza higjene brónquica quando } \\
\text { necessário }\end{array}$ & $130 / 131(99,2)$ & $24 / 25(96,0)$ & 1,08 & $(0,95-1,12)$ & $0,296^{*}$ \\
\hline $\begin{array}{l}\text { Usa EPI durante higiene } \\
\text { brónquica }\end{array}$ & $130 / 131(99,2)$ & $25 / 25(100,0)$ & 0,99 & $(0,98-1,00)$ & $1,000^{*}$ \\
\hline $\begin{array}{l}\text { Realiza higjene brönquica com } \\
\text { técrica asséptica }\end{array}$ & $99 / 128(77,3)$ & $21 / 25(84,0)$ & 0.92 & $(0,75-1,11)$ & 0,446 \\
\hline $\begin{array}{l}\text { Seque a sequência tubo-nariz - } \\
\text { boca durante o procedimento de } \\
\text { higiene brönquica }\end{array}$ & $131 / 131(100,0)$ & $23 / 25(92,0)$ & 0.87 & $(0,77-0,99)$ & $0,025^{*}$ \\
\hline $\begin{array}{l}\text { Verifica pressão do culf a cada } \\
\text { periodo }\end{array}$ & $525 / 1172(448)$ & $11 / 31(35,5)$ & 0,86 & $(0,66-1,11)$ & 0,299 \\
\hline Mantém pressăo do cuffadequada & $87 / 528(16,5)$ & $1 / 1(9,1)$ & 1.81 & $(0,27-11,86)$ & $1.000^{*}$ \\
\hline $\begin{array}{l}\text { Avalia radiografa após a instalaçäo } \\
\text { da sonda nasoenteral }\end{array}$ & $05 / 76(6,6)$ & $2 / 7(28,6)$ & 0,23 & $(0,05-0,98)$ & $0,105^{*}$ \\
\hline
\end{tabular}

FTosta do Fishor 


\section{Estratégias educativas para profilaxia da PAV}

Em relação aos cuidados com o manuseio do VM, a estratégia educativa apresentou eficácia de $43 \%$ para o grupo de intervenção $(p=0,048)$. Durante o período da intervenção, tanto os participantes do grupo intervenção quanto do grupo de comparação que realizaram procedimentos fora das recomendações foram orientados pela gerência do setor e pelos membros do Serviço de Controle de Infecção Hospitalar (SCIH) sobre a importância da técnica asséptica na montagem do VM.

Em relação ao item higiene da língua, a eficácia da estratégia educativa foi de $51 \%(p=0,043)$. Contudo, o uso do digluconato de clorexidina apresentou eficácia de $21 \%$, mas não significativa $(p=0,48)$.

No item sequência correta da higiene brônquica, a eficácia correspondeu a 13\% ( $p=0,006)$. Nos demais aspectos avaliados desse procedimento, a intervenção não foi capaz de mudar significativamente o comportamento dos profissionais no período do estudo.

A troca de nebulizador, a mudança de decúbito, o uso de antisséptico bucal, a verificação e a manutenção de pressão de cuffadequada parecem ter sofrido algum efeito da intervenção, mas não foram significativos.

\section{DISCUSSÃO}

Neste estudo observou-se que vários participantes apresentaram qualificação superior à exigida pela função. Essa particularidade pode contribuir para que eles tomem decisões e exerçam atividades que apresentam maior risco e são de competência privativa do enfermeiro ${ }^{11,12,13}$, fato que foi registrado durante a coleta de dados.

No presente estudo foi possível evidenciar melhor eficácia do workshop como estratégia de educação continuada para higiene da língua e montagem do ventilador com técnica asséptica.

A melhor eficácia do procedimento de higienização da língua pode ser explicada pelo fato de o workshop ter abordado o assunto por maior tempo e/ou de ter sido oferecida prática individualizada. Todavia, a baixa eficácia da estratégia educativa para o uso da clorexidina após higiene bucal pode ter sido influenciada pela relutância de alguns profissionais devido ao sabor da solução. Os profissionais que manifestaram essa preocupação durante os workshops, coincidentemente, foram reforçados pelas discussões na mídia nacional que, na época, ressaltou aspectos negativos do seu uso. Além disso, houve problemas com a recomendação de uso do produto de manutenção por 60 segundos na cavidade bucal, preferencialmente aplicado com dedo de luva e ou com gazes sem aspirar: alguns pacientes não apresentam abertura mandibular suficiente para execução correta desta etapa do procedimento. A mudança nos cuidados com a higiene lingual evidenciada no estudo é benéfica para a redução da colonização da placa dental9,14.

A menor eficácia da estratégia do workshop para a sequência correta da higiene brônquica provavelmente ocorreu por ser um procedimento realizado corretamente por quase todos os profissionais de ambos os grupos. É possível que, em locais onde o uso de sequência correta para a higiene brônquica seja um problema, o workshop possa apresentar melhores resultados. A falta de resultados mais positivos relativos à periodicidade da higiene brônquica e uso de EPI durante 0 procedimento provavelmente também seja devida à realização adequada por quase todos os profissionais de ambos os grupos. Assim, há dificuldade na comparação entre estudos realizados em outros contextos e com outros desfechos. Por exemplo, um estudo conduzido na Tailândia usou método autodirigido, cartazes e fichas técnicas com repetição a cada seis meses por quatro anos com uma população de enfermeiros e terapeutas respiratórios, apresentou redução de 59\% $(p<0,001)$ de PAV 15 .

Os resultados relativos a outros cuidados como a mobilização no leito, a elevação da cabeceira a 30 graus ou mais, a manutenção da técnica asséptica durante 0 procedimento de aspiração traqueal, a indicação adequada da aspiração traqueal pelos técnicos de enfermagem, o uso de EPI durante a aspiração traqueal e a frequência da verificação da pressão de cuff podem ser considerados positivos mesmo sem significância estatística, desde que outros estudos mostraram necessidade de investir em educação continuada por período prolongado para que resultados positivos possam ser detectados. De fato, alguns resultados positivos são obtidos após intervenção educativa prolongada ${ }^{16}$.

Apesar do aumento da frequência de verificação da pressão intracuff, os valores ainda foram inadequados, diferente dos resultados de outros estudos que instituíram prática de mensuração e programa de treinamento trimestral dos trabalhadores, enfocando os malefícios da utilização da pressão do cuff inadequada e diminuição da ocorrência de complicações da inadequação da pressão do cuff ${ }^{7}$.

Uma condição específica do hospital em questão pode ter contribuído para a baixa eficácia da estratégia educativa para avaliação da radiografia após a instalação da sonda nasoenteral. Apesar das recomendações ${ }^{18} \mathrm{e}$ da ênfase durante os workshops, a instituição não possibilita a realização de uma radiografia a cada instalação ou troca da sonda nasoenteral. Esse fato foi considerado uma limitação do estudo.

Considera-se como outra limitação do estudo a não randomização, como fator contribuinte da redução da validade externa dos resultados apresentados. A não randomização provavelmente ainda afetou a avaliação de alguns procedimentos menos incidentes.

\section{CONCLUSÕES}

Os resultados mostram que a intervenção educativa teve eficácia para a realização correta da montagem do VM com técnica asséptica, a higienização da língua e a manutenção da ordem correta tubo-nariz-boca durante 0 procedimento de higiene brônquica. Considerando que o 
workshop foi utilizado associado a cartazes com charges, e com a expectativa de que a aprendizagem contínua transforme a prática, são necessários novos estudos com programas periódicos de educação associados à auditoria diária, a fim de avaliar e testar diferentes formas de intervenção educativas.

Além disso, sugere-se a realização de novos estudos para confirmar os resultados aqui apresentados.

\section{REFERÊNCIAS}

1.Koenig S, Truwit JD. Ventilator-associated pneumonia: diagnosis, treatment and prevention. Clin Microbiol Rev. [on-line] 2006; [cited 2010 Apr 18]; 19(4): [aprox 20 telas]. Available from: http:// www.ncbi.nlm.nih.gov/pmc/articles/PMC1592694/pdf/0051-05.pdf

2.Toufen Jr C, Hovnanian ALD, França SA, Carvalho CRR. Prevalence rates of infection in intensive care units of a tertiary teaching hospital. Rev Hosp Clin Fac Med S Paulo. [on-line] 2003; [cited 2011 out 04]; 58(5): [aprox. 5 telas]. Available from: http://www.scielo.br/pdf/rhc/ v58n5/v58n5a04.pdf.

3.Pinaa E, Ferreira E, Marques A, Matos B. Infecções associadas aos cuidados de saúde e segurança do doente. Rev Port Saude Publica. [on-line] 2010; [citado 2010 mar 12]; (10): [aprox. 12 telas]. Disponível em: http:// www.ensp.unl.pt/dispositivos-de-apoio/cdi/cdi/sector-depublic a coe s/revist a / $2010 / \mathrm{pdf} / \mathrm{vol}$ u m e t e matico-seguranca-do-doente/4Infeccoes $\% 20$ as s o ciadas $\% 20$ a os $\% 20$ cuidados $\%$ 20 de $\% 20$ saude $\% 20$ e\%20seguranca $\% 20$ do\%20doente.pdf

4.Agência Nacional de Vigilância Sanitária-ANVISA. Unidade de Investigação e Prevenção das Infecções e dos Eventos Adversos. Gerência Geral de Tecnologia em Serviços de Saúde- GGTES. Infecções do trato respiratório: orientações para prevenção de infecções relacionadas à assistência à saúde. [on-line)] 2009; [citado 2010 mar 12]; [aprox 27 telas] Disponível em:http://www.anvisa.gov.br/ servicosaude/controle/manual_\%20trato_respirat\%F3rio.pdf

5.Sociedade Brasileira de Pneumologia e Tisiologia- SBPT. Diretrizes brasileiras para tratamento das pneumonias adquiridas no hospital e das associadas à ventilação mecânica. J Bras Pneumol. [on-line] 2007; [citado 2011 ago 12]; 33(supl 1): [aprox 30 telas]. Disponível em: http://www.scielo.br/pdf/jbpneu/v33s1/01.pdf

6.Tablan 0, Anderson L, Besser R, Bridges C, Hajjeh R. Guidelines for preventing healthcare-associated pneumonia 2003: recommendations of $C D C$ and the Healthcare Infection Control Practices Advisory Committee- MMWR. [on-line] 2004; [cited 2010 Mar 12]; 53(RR-3): [aprox. 36 telas]. Available from: http://www.cdc.gov/hicpac/pdf/ guidelines/HApneu2003guidelines.pdf
7.Agência Nacional de Vigilância Sanitária- ANVISA. Unidade de Investigação e Prevenção das Infecções e dos Eventos Adversos. Gerência Geral de Tecnologia em Serviços de Saúde- GGTES. Trato respiratório: critérios nacionais de infecções relacionadas à assistência à saúde. [on-line] 2009; [citado 2011 nov 25]; [aprox 34 telas]. Disponível em: http://www.cve.saude.sp.gov.br/htm/ih/ pdf/manual_trato_respiratorio.pdf

8.Klompas M. Prevention of ventilator-associated pneumonia. Expert Rev Anti Infect Ther. [on-line] 2010; [cited 2011 Nov 25]; 8(7): [aprox 9 telas]. Available from: http://www.expert-reviews.com/doi/ pdf/10.1586/eri.10.59.

9.Baxter AD, Jill A, Bedard J, Malone-Tucker S, Slivar S, Langill M, at al. Adherence to simple and effective measures reduces the incidence of ventilator-associated pneumonia. Can J Anesth. [online] 2005; [cited 2011 0ct 19]; 52(5): [aprox 6 telas]. Available from: http://www.springerlink.com/content/u21111342r845v0u/ fulltext.pdf.

10.Freire IL. A ventilação mecânica invasiva e a intervenção da equipe de saúde na prevenção das pneumonias nosocomiais [mestrado]. Natal: Escola de Enfermagem,Universidade Federal Rio Grande do Norte; 2005a.

11.Conselho Federal de Enfermagem(BR). Lei n. 7.498, de 25 de junho de 1986. Dispõe sobre a regulamentação do exercício da enfermagem e dá outras providências. Diário Oficial da Republica Federativa do Brasil, Brasilia, DF, 26 jun 1986.

12.Endotracheal suctioning of mechanically ventilated patients with artificial airways. Respiratory Care. [on-line]. 2010; [cited 2011 0ct 26]; 55(6): [aprox 6 telas]. Available from: http://www.rcjournal.com/ cpgs/pdf/06.10.0758.pdf

13.American Association of Critical Care Nurses-AACN. Practice Alerts. Oral care for patients at risk for ventilator- associated pneumonia. [on-line] 2010; [cited 2012 Mar 1]: [aprox 3 telas]. Available from: http://www.aacn.org:88/WD/Practice/Docs/PracticeAlerts/ oral\%20care\%2004-2010\%20final.pdf

14.Tantipong H, Morkchareonpong C, Jaiyindee S, Thamlikitkul V. Randomized controlled trial and meta- analysis of oral decontamination with $2 \%$ chlorhexidine solution for the prevention of ventilatorassociated pneumonia. Infection Control and Hospital Epidemiology. [on-line] 2008; [cited 2012 Mar 12]; 29(2): [aprox 5 telas]. Available from: http://www.jstor.org/stable/10.1086/526438

15.Apisarnthanarak A, Pinitchai U, Thongphubeth K, Yuekyen C, Warren DK, Zack JE et al. Effectiveness of an educational program to reduce ventilator-associated pneumonia in a tertiary care center in Thailand: a 4-year study. Clin Infect Dis. [on-line] 2007; [cited 2012 Mar 11]; 45(6): [aprox. 7 telas]. Available from: http://www.ncbi.nlm.nih.gov/ pubmed/17712753.

16.Bouadma L, Deslandes E, Lolom I, Le Corre B, Mourvillier B, Regnier, et al. Long-term impact of a multifaceted prevention program on ventilator-associated pneumonia in a medical intensive care unit. Clin Infect Dis. [on-line] 2010; [cited 2011 0ct 19]; 51(10): [aprox.7 telas]. Available from: http://www.ncbi.nlm.nih.gov/pubmed/ 20936973. 


\section{Estratégias educativas para profilaxia da PAV}

17.Juliano SRR, Juliano MCR, Cividanes JP, Houly JGS, Gebara OCE, Cividanes GVL, et al. Medidas dos níveis de pressão do monitorização em Unidade de Terapia Intensiva: considerações sobre os benefícios do treinamento. Rev Bras Ter Intensiva. [on-line] 2007; [citado 2011 out 25]; 19(3): [aprox 4 telas]. Disponível em: http://www.scielo.br/ $\mathrm{pdf} / \mathrm{rbti} / \mathrm{v} 19 \mathrm{n} 3 / \mathrm{v} 19 \mathrm{n} 3 \mathrm{a} 08$.pdf.

18.Westphal FLLCdL, Lima Netto JC, Silva MDSD, Freitas CAMF, Lima ILDQ, Westphal DC. Complicações torácicas por sonda de Dobb-Hoff. E- JCT. [on-line] 2012; [citado 2012 jun 19]; 1(1): [aprox 4 telas]. Disponível em: http://www.sbct.org.br/pdf/e_jbct/edicao_1/ artigo_3.pdf 